identified differential expression of approximately 2000 genes between 564 and WT mice. To identify a role for interferon, RNA libraries were prepared from 564 and WT mice treated with a neutralizing antibody to IFNAR. Of the differentially expressed genes in lupus mice, about 20\% were IFNa dependent. Notably, the microglia isolated from the lupus strain expressed an interferon gene signature that included IFNbeta but not IFNa. Pathway analysis of the interferon-dependent genes identified increased expression of genes important in phagocytosis and metabolic activity. Finally, reduction in peripheral levels of interferon alpha was protective from synapse loss and altered behavior (Bialas et al Nature 2017).

Conclusions We conclude that elevated blood levels of IFNa can promote neurological symptoms in mice. These findings suggest that therapies that block peripheral autoimmunity and reduce circulating levels of IFNa may protect against the symptoms of CNS lupus.

Acknowledgements Supported by grants from The Lupus Research Alliance (USA) and National Institutes of Health (USA).

\section{TD-07 DISSECTING THE ROLE OF MYELOID CELLS IN LUPUS NEPHRITIS}

${ }^{1,2}$ Paul J Hoover*, ${ }^{1}$ Tony D Jones, ${ }^{2}$ Karen H Costenbader, ${ }^{1}$ Nir Hacohen. ${ }^{1}$ Broad Institute of MIT and Harvard, Cambridge MA, USA; ${ }^{2}$ Division of Rheumatology, Allergy, Immunology, Department of Medicine, Brigham and Women's Hospital, Harvard Medical School, Boston MA USA

\subsection{6/lupus-2018-Ism.126}

Background The NIH- sponsored AMP -PEARL (Accelerating Medicine Partnership lupus network Pathway Exploration and Analysis in RenaL disease) consortium sequenced RNA from 2900 single cells from lupus nephritis kidney biopsies from 24 patients and discovered 22 immune cell types. While providing unprecedented molecular information, the study lacked in situ immune cell spatial context and was underpowered for associations with clinical outcomes. Thus, we aimed to investigate the spatial organization of the immune landscape in lupus nephritis tissue and determine whether in situ immune cell organization drives clinical outcomes. We hypothesize that the immune cell infiltrate in lupus nephritis tissue is highly organized and promotes kidney remodeling that drives clinical outcomes.

Methods We assembled a new, larger cohort of 40 lupus nephritis patients who presented with varying disease severity, undergoing their first kidney biopsies (naïve to potent immune-modulators). From these biopsy samples, we are dissecting the organizational landscape, role, and clinical associations of the five newly discovered myeloid subsets in formalin fixed paraffin embedded tissue. To map the in situ spatial relationships, we converted single cell RNA-sequencing signatures into molecular stains based on highly expressed myeloid subset-specific discriminatory genes with known biological functions. We then stained clinical samples from our cohort for multiplex fluorescent imaging of newly identified cell subsets.

Results We have validated the five new myeloid subsets (three monocytes, one dendritic cell, and one macrophage) in class IV lupus nephritis tissue and we are working to do the same in other histological classes. In addition, we are deconstructing myeloid subset cellular neighborhoods by mapping each to renal compartments, tissue damage, and quantifying the composition of the neighborhood's immune cell (T, B, NK cells) infiltrate.

Conclusions By converting single cell RNA sequencing information into molecular stains we have developed a novel approach to validate in situ five newly identified myeloid subsets in class IV lupus nephritis and we are pursuing other histological classes. We are deconstructing the in situ cellular neighborhood by mapping each subset across tissue to determine its relation to histopathological lesions and clinical outcomes. We expect these data to link the new myeloid subsets to distinct cellular neighborhoods, and provide the first-ever connection between well-characterized in situ local immune responses, their histopathologic lesions, and clinical outcomes in our clinically annotated cohort. This work will lay the groundwork for disease re-classification based on the immune response and could highlight important cell-types and pathways driving disease for follow up studies.

\section{TD-08 HIGH TYPE I INTERFERON ACTIVITY IS ASSOCIATED WITH ACTIVE CLASS III/IV LUPUS NEPHRITIS IN EUROPEAN-AMERICAN LUPUS PATIENTS INDEPENDENT OF ANTI-DSDNA ANTIBODIES}

${ }^{1}$ Taro Iwamoto, ${ }^{2}$ Jessica M Dorschner, ${ }^{1}$ Mark A Jensen, ${ }^{2}$ Danielle Vsetecka, ${ }^{2}$ Shreyasee Amin, ${ }^{2}$ Ashima Makol, ${ }^{2}$ Floranne C Ernste, ${ }^{2}$ Thomas Osborn, ${ }^{2}$ Kevin Moder, ${ }^{2}$ Vaidehi Chowdhary, ${ }^{1}$ Timothy B Niewold*. ${ }^{1}$ Colton Center for Autoimmunity, New York University, New York, NY, USA; ${ }^{2}$ Mayo Clinic College of Medicine, Rochester, MN, USA

\subsection{6/lupus-2018-Ism. 127}

Background Lupus nephritis (LN) is one of the most severe types of organ involvement in systemic lupus erythematosus (SLE), despite the recent advances in immunosuppressive therapies. High type I interferon (IFN) is a heritable risk for SLE, and some previous studies have suggested a link between high IFN and lupus nephritis. However, little is known about the relationships between high levels of IFN and the subtypes of LN, and whether IFN is more associated with anti-dsDNA antibodies or with clinical nephritis.

Methods We studied 244 European-American (EA) SLE patients and measured type I IFN in sera by performing WISH IFN bioassay as described previously. Subtypes of LN were confirmed by renal biopsy review. Complements, antidsDNA and other auto-antibodies were measured in the clinical laboratory, and standard clinical cut-offs were used to define a positive result. Non-parametric analyses were used to compare IFN data with the clinical data. RNA in-situ hybridization was used to assess markers of plasmacytoid dendritic cells (PDCs) and IFN-induced gene expression in renal biopsy samples.

Results IFN level and SLEDAI score was positively correlated $(\mathrm{r}=0.26, \mathrm{p}<0.0001$, Spearman $)$ in our cross-sectional evaluation. EA subjects with a high level of IFN (IFN score $\geq 2$ ) were more likely to have renal manifestations compared to the subjects with a low level of IFN (IFN score <2) $(\mathrm{p}<0.001, \mathrm{OR}=3.0$, Fisher's exact test). In addition, the incidence rate of class III/IV LN was significantly higher among patients with a high level of IFN compared to the patients with low levels of IFN ( $<<0.01, \mathrm{OR}=5.5$, Fisher's exact test). Notably, IFN level was significantly higher in active class III/ IV LN compared to inactive class III/IV LN $(p<0.05$ MannWhitney U) and this was not observed in non-class III/IV LN populations. Positivity of ds-DNA antibody did not show significant difference between inactive class III/IV LN and active 